ACCEPTED MANUSCRIPT

\title{
Tuning the thermoelectric response of silicene nanoribbons with vacancies
}

To cite this article before publication: Cesar D Núñez et al 2020 J. Phys.: Condens. Matter in press https://doi.org/10.1088/1361-648X/ab7e56

\section{Manuscript version: Accepted Manuscript}

Accepted Manuscript is "the version of the article accepted for publication including all changes made as a result of the peer review process, and which may also include the addition to the article by IOP Publishing of a header, an article ID, a cover sheet and/or an 'Accepted

Manuscript' watermark, but excluding any other editing, typesetting or other changes made by IOP Publishing and/or its licensors"

This Accepted Manuscript is @ 2020 IOP Publishing Ltd.

During the embargo period (the 12 month period from the publication of the Version of Record of this article), the Accepted Manuscript is fully protected by copyright and cannot be reused or reposted elsewhere.

As the Version of Record of this article is going to be / has been published on a subscription basis, this Accepted Manuscript is available for reuse under a CC BY-NC-ND 3.0 licence after the 12 month embargo period.

After the embargo period, everyone is permitted to use copy and redistribute this article for non-commercial purposes only, provided that they adhere to all the terms of the licence https://creativecommons.org/licences/by-nc-nd/3.0

Although reasonable endeavours have been taken to obtain all necessary permissions from third parties to include their copyrighted content within this article, their full citation and copyright line may not be present in this Accepted Manuscript version. Before using any content from this article, please refer to the Version of Record on IOPscience once published for full citation and copyright details, as permissions will likely be required. All third party content is fully copyright protected, unless specifically stated otherwise in the figure caption in the Version of Record.

View the article online for updates and enhancements. 


\title{
Tuning the thermoelectric response of silicene nanoribbons with vacancies
}

\author{
C. Núñez, ${ }^{1}$ M. Saiz-Bretín, ${ }^{2}$ P. A. Orellana, ${ }^{1}$ L. Rosales, ${ }^{1}$ and F. Domínguez-Adame ${ }^{2}$ \\ ${ }^{1}$ Departamento de Física, Universidad Técnica Federico Santa María, Casilla 110 V, Valparaíso, Chile \\ ${ }^{2}$ GISC, Departamento de Física de Materiales, Universidad Complutense, E-28040 Madrid, Spain
}

(Dated: February 17, 2020)

\begin{abstract}
In this work, we present a thorough study of the thermoelectric properties of silicene nanoribbons in the presence of a random distribution of atomic vacancies. By using a linear approach within the Landauer formalism, we calculate phonon and electron thermal conductances, the electric conductance, the Seebeck coefficient and the figure of merit of the nanoribbons. We found a sizable reduction of the phonon thermal conductance as a function of the vacancy concentration over a wide range of temperature. At the same time, the electric properties are not severely deteriorated, leading to an overall remarkable thermoelectric efficiency. We conclude that the incorporation of vacancies paves the way for designing better and more efficient nanoscale thermoelectric devices.
\end{abstract}

PACS numbers: $65.80 .-\mathrm{g}, 72.80 . \mathrm{Ng}, 73.63 .-\mathrm{b}$

\section{INTRODUCTION}

Thermoelectric efficiency is achieved when the dimensionless figure of merit $Z T=S^{2} \sigma T / \kappa$ is high enough. ${ }^{1}$ In the definition of the figure of merit, $S$ stands for the Seebeck coefficient, and $\sigma$ and $\kappa$ are the electric and thermal conductances at a temperature $T$, respectively (see, e.g., Ref. 2). For instance, values of $Z T$ larger than unity represent a heat-to-electricity conversion efficiency larger than $10 \%$. Current thermoelectric materials display efficiency in the range of $5 \%$ to $20 \%$. Thus, efficient thermoelectric devices demand materials with strongly suppressed thermal conductance to create a large temperature gradient but still high electronic conduction to minimize Joule heating. Both electrons and phonons contribute to heat current and, consequently, thermal conductance can be split as $\kappa=\kappa_{\mathrm{el}}+\kappa_{\mathrm{ph}}$. Strategies for enhancing thermoelectric efficiency seek to reduce both contributions simultaneously without affecting electronic conduction. In conventional metals, however, the classical Wiedemann-Franz law imposes a limit because the ratio $\sigma T / \kappa_{\mathrm{el}}$ is a universal constant. Therefore, a decrease of $\kappa_{\mathrm{el}}$ is accompanied by a concomitant decrease of $\sigma$ in bulk metals. Hence, reducing the lattice thermal conductance $\kappa_{\mathrm{ph}}$ by increasing phonon scattering is one of the most promising routes to improve thermoelectric materials. Approaches such as nanostructuring, nanocomposites and doping are found to enhance the thermoelectric efficiency by simultaneously tuning various properties of materials and, in particular, enhancing phonon scattering (see Refs. 3 and 4 for recent reviews).

In the last few years, several works have provided theoretical $^{5-8}$ and experimental evidence ${ }^{9-12}$ that nanostructuring yields thermoelectric efficiency unachievable with bulk materials. On the one hand, quantum effects allow thermoelectric devices to overcome the limitations arising from the classical Wiedemann-Franz law. Nanodevices with sharp resonances in the electron transmission (such as Fano lineshapes) are good candidates for highly efficient heat-to-electricity converters because the ratio $\sigma T / \kappa_{\mathrm{el}}$ increases well above the classical Wiedemann-
Franz limit. ${ }^{13-19}$ On the other hand, nanometer-sized objects exhibit a reduced lattice thermal conductance due to an increased phonon scattering. ${ }^{20-23}$ Thus, nanostructuring facilitates achieving large $Z T$ and, consequently, more efficient thermoelectric devices like refrigerators and generators. ${ }^{24}$

Nanostructured graphene, such as nanoribbons and nanorings, stand out because of the straightforward way in which they exploit quantum interference effects. Recently, we demonstrated theoretically that graphene nanorings might be useful as thermoelectric devices. ${ }^{25}$ Thermal conductance can be greatly reduced in graphene nanoribbons by rough edges, ${ }^{26}$ hydrogen-passivation ${ }^{27}$ and patterning. ${ }^{28-32}$ Unfortunately, lattice heat conduction, which is expected to be the most important contribution to heat transport in carbon materials due to the strong covalent $s p^{2}$ or $s p^{3}$ bonding, is still large for thermoelectric applications. Thermal conductivity in bulk graphene is as high as $2000-4000 \mathrm{~W} / \mathrm{m} \mathrm{K}$ at room temperature ${ }^{33}$ but it is largely reduced in narrow nanoribbons $(150 \mathrm{~W} / \mathrm{m} \mathrm{K})$ and even more in nanorings $(100 \mathrm{~W} / \mathrm{m} \mathrm{K})$ due to scattering of lattice vibration modes at the bends. ${ }^{23}$

Bulk silicene, the silicon analog of graphene, exhibits an in-plane thermal conductivity of $20 \mathrm{~W} / \mathrm{m} \mathrm{K}$ at room temperature, according to equilibrium molecular dynamics simulations ${ }^{34}$ (see Ref. 35 for a review on recent advances in silicene). This value is one order of magnitude lower than that of bulk silicon. Graphene and silicene share a common honeycomb lattice structure, but the larger ionic radius of silicon compared to carbon induces a buckling of the lattice. ${ }^{36,37}$ Buckling has an impact on the vibrational modes of silicene that undergo phonon softening and stiffening, thus reducing phonon heat conduction. Besides, in these systems, the presence of point defects such as monovacancies and divacancies, generate a significant diminution of the thermal conductance of silicene sheets owing to phonon-defect scattering. ${ }^{34}$

In this paper, we study the thermal and thermoelectric properties of silicene nanoribbons (SNRs) at low temperatures. We have calculated the thermal conductance $\kappa_{\mathrm{ph}}$ 
when a random distribution of point defects (such as vacancies) in a diluted regime is created in the SNR. We have then focused on the impact of vacancies concentration on the phonon thermal conductance of SNRs at different temperatures. We have found a sizable reduction of $\kappa_{\mathrm{ph}}$ as a consequence of the reduced phonon mean free path. This decrease depends on the defect concentration and temperature, especially in narrow SNRs. Furthermore, we have observed that the presence of vacancies leads to an enhancement of the Seebeck coefficient, which is mainly due to the quantum interference phenomena within the device. These features affect the thermoelectric conversion efficiency factor and, most importantly, enhanced values of $Z T$ arise at low defects concentration. In this sense, we suggest that point defects, such as monovacancies, divancancies, or ad-atoms, might improve the thermoelectric behavior of SNR by exploiting interference effects and reducing the phonon mean free path.

\section{THEORETICAL MODEL}

The system under study consists of a rectangular SNR of width $W$ and length $L$ connected to the source and drain leads, as shown schematically in Fig. 1. In order to prevent topologically protected edge states that appear at the Fermi energy in zig-zag nanoribbons, we restrict ourselves to nanoribbons with fully hydrogen saturated armchair edges (A-SNR) hereafter. The electronic properties of these ribbons have been widely studied by using both DFT calculations and single-band tightbinding (TB) Hamiltonian. ${ }^{35,38-41}$ Both approximations give reasonable quantitative (DFT) and qualitative (TB) descriptions of the physical properties of SNR. In these approaches, hydrogen saturated A-SNR behaves as semiconductor or semi-metallic, depending on the number of silicon dimers along the transverse direction of the ribbon. Thus, for widths $W=3 p$ and $W=3 p+1$ ( $p$ being an integer number), A-SNRs behave as semiconductors with a decreasing electronic gap as $W$ increase; whereas for $W=3 p-1$ A-SNRs behave as semi-metallic, with a zero energy gap at the charge neutrality point. ${ }^{40,41}$

In this context, and by adopting the single band TB framework, we have considered a random distribution of vacancies in the central region of a semi-metallic ribbon, which are represented with translucent circles in Fig. 1. From the numerical point of view, for the electronic part of the calculations, the way to simulate vacancies in the lattice is by setting large on-site energy, around $10^{4}$ times the energy scale of the A-SNR. It prevents electrons from occupying those sites. On the other hand, for the phonon thermal conductance calculations, we randomly removed atoms in the lattice by setting zero to the inter-atomic potential between the vacancy and the four nearest-neighbor atoms.

In order to study the thermal transport of the ribbons, we have assumed the linear response approximation tak-

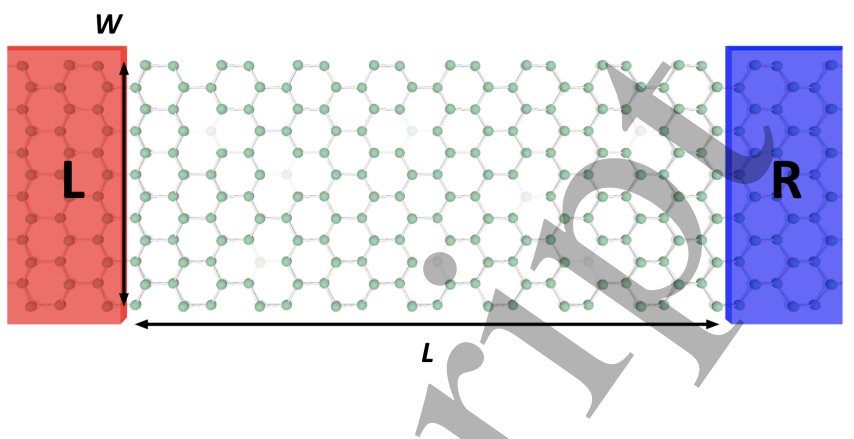

FIG. 1. Schematic view of the device. The central A-SNR is connected to left $(\mathrm{L})$ and right $(\mathrm{R})$ leads of the same material. The translucent circles represent the atomic vacancies on the nanoribbon.

ing into account a small temperature difference between both leads, $T_{L}$ and $T_{R}$, respectively (with $T_{L}>T_{R}$ ). In our study, we can neglect electron-phonon and phononphonon interactions ${ }^{42,43}$ within the conductor because these interactions are of higher order in comparison to the harmonic interaction term, which we have used to describe qualitatively ballistic thermal transport. In this approximation, phonon transport can be calculated similarly as the electronic counterpart. Thus, it is necessary to solve the eigenvalue equation $K \psi(r)=\omega^{2} M \psi(r)$, where $M$ is the diagonal mass matrix and $K$ is the dynamical matrix of the system, which is calculated by using the finite difference method. Matrix elements of $K$ are given by $K_{i \alpha, j \beta}=d F_{i \beta} / d r_{i \alpha}$ where $F_{j \beta}$ is the force on the $j$-th atom in the $\beta$ Cartesian direction due to the displacement of the $i$-th atom in the $\alpha$ Cartesian direction. In order to calculate the interatomic forces, one needs to know the interaction potential, which is here parameterized by a semi-empirical potential. In our case, we have chosen the well-known Stillinger-Weber potential. ${ }^{44}$ Under these assumptions, Landauer's formulation is flawless and reliable for calculating phonon transport properties. ${ }^{45}$ The key magnitude then is the ribbon width dependent phonon transmission $\mathcal{T}_{N}(\omega)$ through the A-SNR (where $N$ stands to the number of Si-atoms dimers along the ribbon width) that will be addressed by the non-equilibrium Green's functions (NEGF) method. This formalism is a useful and powerful method to study dynamical processes in non-equilibrium many-body systems. To this end, the system is split into three spatial regions, namely left contact, scattering region (conductor), and right contact. Landauer's approach makes no distinction between fermions and bosons, and, as a consequence, transport equations are essentially the same in both cases, except for the occupation functions of the leads.

In this regard, the thermal current of phonons at a temperature $T$ can be calculated from the transmission function $\mathcal{T}_{N}(\omega)$ as follows ${ }^{46,47}$

$$
J_{\mathrm{ph}}=\frac{1}{2 \pi} \int_{0}^{\infty} \hbar \omega \mathcal{T}_{N}(\omega)\left[n_{B}\left(T_{L}\right)-n_{B}\left(T_{R}\right)\right] \mathrm{d} \omega
$$


where $n_{B}(T)=1 /\left(e^{\hbar \omega / k_{B} T}-1\right)$ is the phonon occupation number at a given temperature $T$, and $T_{L}=$ $T+\Delta T / 2$ and $T_{R}=T-\Delta T / 2$. In the linear regime, when $\Delta T$ is small, the phonon contribution to the thermal conductance of the A-SNR, $\kappa_{\mathrm{ph}}(T)=J_{\mathrm{ph}} / \Delta T$, is given as

$$
\kappa_{\mathrm{ph}}(T)=\frac{\hbar^{2}}{2 \pi k_{B} T^{2}} \int_{0}^{\infty} \omega^{2} \mathcal{T}_{N}(\omega) \frac{e^{\hbar \omega / k_{B} T}}{\left(e^{\hbar \omega / k_{B} T}-1\right)^{2}} \mathrm{~d} \omega .
$$

This expression holds in the ballistic regime since it is assumed that $\mathcal{T}_{N}(\omega)$ includes only the coherent part of the transmission. For this purpose, we used QuantumATK 2017.0. ${ }^{48}$ Phonon-phonon interaction is the major limiting factor of the thermal conductance at high temperatures $\left(T>\Theta_{\mathrm{S}}\right.$, where $\Theta_{\mathrm{S}} \simeq 640 \mathrm{~K}$ is the Debye temperature in bulk silicene ${ }^{49}$ ) and it is not entering into the calculation of $\mathcal{T}_{N}(\omega)$. Therefore, Eq. (2) will overestimate the value of the thermal conductance at high temperature. Phonon-phonon interaction could be included through a non-linear fit of the self-energy in the NEGF equations, or they can be treated by the Boltzmann transport theory if needed. The main advantage of Landauer's formulation (besides its computational speed in comparison to other approaches) is that no further approximations are needed. It is worth mentioning that all curves presented in this work are averaged over a hundred random realizations of disorder, avoiding any spurious results originated from statistical fluctuations.

\section{RESULTS}

We start our analysis by considering the confinement effects on the phonon thermal transport properties of the considered A-SNR. In Fig. 2, we show the phonon thermal conductance $\kappa_{\text {ph }}$ as a function of temperature for different values of the number of dimers $N$ along the transverse direction of the ribbon. As expected, the thermal conductance $\kappa_{\mathrm{ph}}$ shows higher values as the ribbon width is increased because the number of allowed phonon modes available for heat transport increases. ${ }^{50,51}$ The thermal conductance at temperatures lower than $100 \mathrm{~K}$ takes values in the range $0.1-0.5 \mathrm{nW} / \mathrm{K}$ while at higher temperatures, the value of the $\kappa_{\mathrm{ph}}$ saturates. This trend is due to the activation of different phonon modes as temperature increases. At low temperature (lower than $100 \mathrm{~K}$ ) only longitudinal and transverse acoustic phonon modes contribute to the thermal conductance. ${ }^{50}$ As soon as the temperature is increased, but still in the ballistic regime condition, all phonons of the optical branches and mostly the long-wavelength acoustic modes contribute to $\kappa_{\mathrm{ph}}$ and the conductance reaches a constant value. It is worth mentioning that anharmonic terms, such as phonon-phonon and electron-phonon interactions, are always present in the system; however, at temperatures lower than $T>\Theta_{\mathrm{S}}=640 \mathrm{~K}$ these effects play a minor role and are considered perturbations of the second or- der. At higher temperatures, they must be taken into account, because the anharmonicity of these terms dominates the thermal response of the sample. In that case, the ballistic regime is no longer valid, and the calculated phonon thermal conductance is overestimated.

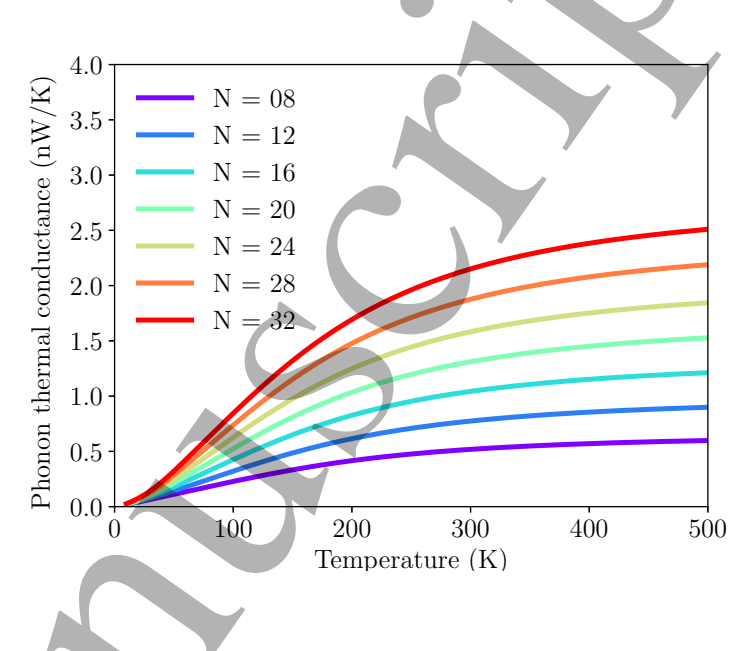

FIG. 2. Phonon thermal conductance as a function of temperature for different A-SNR widths and for a length $L \simeq 26 \mathrm{~nm}$.

C

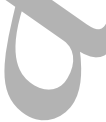

A. Impact of vacancies on the thermal conductance

During growth, synthesis, and physical manipulation of materials at the nanoscale, it is common to observe the occurrence of different kinds of defects in the nanostructure, such as dislocations, Stone-Wales defects, vacancies, impurities or adatoms, among others. These defects not only significantly affect the electronic properties of the material, but also act as phonon localization/delocalization centers, with the consequent deterioration of the thermal properties. ${ }^{34,52,53}$ By using firstprinciples calculation, several authors have studied different configurations of defects in silicene, considering single vacancies, groups of vacancies (clusters), or even extended line of defects. ${ }^{54,55}$ In these works, it is shown that due to silicene presents $s p^{3}$ hybridization, the formation energy of these defects is reduced, and consequently, the defective silicene becomes a stable structure. This behavior has been observed in other two-dimensional systems as well, such as graphene, black phosphorous and $\mathrm{MoS}_{2}$ layers. Recent studies have shown that defects can dramatically reduce phonon transport in graphene, ${ }^{56}$ silicene nanowires $^{57}$ and silicene nanoribbons. ${ }^{34,58}$ For instance, Li et al. ${ }^{34}$ have shown that a single vacancy in silicene sheets, of a total of approximately 400 atoms, can diminish the thermal conductance about $78 \%$ in comparison with a pristine sample. These results point out the relevance of vacancies in thermal transport, being a suitable mechanism to reduce the phonon thermal conductance in nanometric systems. 
In this context, in what follows, we focus on the effects of a random distribution of single vacancy defects in a diluted regime, on phonon thermal transport of narrow SNRs. We address the impact of the disorder potential (Anderson-like potential caused by the random distribution of defects) on the phonon thermal transport properties. Despite the fact that single vacancies present higher formation energy per atom in comparison to other defects (such as divacancies or cluster of vacancies), this atomic defect is stable under a wide range of temperatures and, without loss of generality, it represents a convenient way to model a random distribution of defects in the system. It is most important to mention that we have compared the results with those corresponding to divancacy defects, and we have not observed dramatic differences in the general trends of the thermal conductance. Bearing these considerations in mind, we used the Landauer's formulation as given by Eq. (2). Results of the average phonon thermal conductance $\left\langle\kappa_{\mathrm{ph}}\right\rangle$, over a hundred of realizations, of a A-SNR of width $N=20$ dimers and length $L \simeq 26 \mathrm{~nm}$ are shown in Fig. $3 \mathrm{a}$ ), for different concentrations of vacancies $n=0.1 \%, 1 \%, 3 \%$ and $5 \%$. We define the concentration of vacancies $n$ as the ratio between the number of removed atoms and the total number of $\mathrm{Si}$ atoms in the pristine A-SNR and is contained in the dynamical matrix $K$. In order to asséss the reduction of $\kappa_{\mathrm{ph}}$ in the disordered system, for comparison, we have also included the pristine case in this figure.

For all cases, a strong reduction of $\left\langle\kappa_{\mathrm{ph}}\right\rangle$ with respect to the pristine A-SNR is observed in the whole temperature range. The defect-phonon scattering ${ }^{59,60}$ mainly cause this significant decrease. Similar effects have been reported in silicene sheets, where $\kappa_{\mathrm{ph}}$ decreases about $30 \%$ at temperatures greater than $200 \mathrm{~K}$ by the presence of only a single vacancy. ${ }^{34}$ At low temperatures, the long-wavelength acoustic phonons dominate the thermal transport, and $\left\langle\kappa_{\mathrm{ph}}\right\rangle$ takes values lower than to those in pristine samples. Although the curves have the same trend as a function of temperature, it is clear that for concentrations above $1 \%$, the reduction of $\left\langle\kappa_{\mathrm{ph}}\right\rangle$ could be higher than $60 \%$, reaching the saturation limit at temperatures around $300 \mathrm{~K}$. It indicates that vacancies not only reduce the mean-free-path of the optical phonons but also affects the acoustic phonons as well. This behavior is well reflected in the phonon transmission function presented in Fig. $3 \mathrm{~b}$ ). In this plot, it is possible to observe that the acoustic branches (lower energy values) are less affected, in comparison with the optical branches (higher energy values) as the vacancy concentration increases. This can be easily understood because both sub-lattices vibrate almost in phase in an acoustic mode, at least over regions smaller than the phonon wavelength, being less affected at low energy. On the other hand, in the optical branches, the sub-lattices vibrate out of phase. Hence, even for high wavelengths $(k \rightarrow 0)$, optical modes are sensitive to scales of the order of the lattice parameter and, therefore, they are more affected by the disorder.
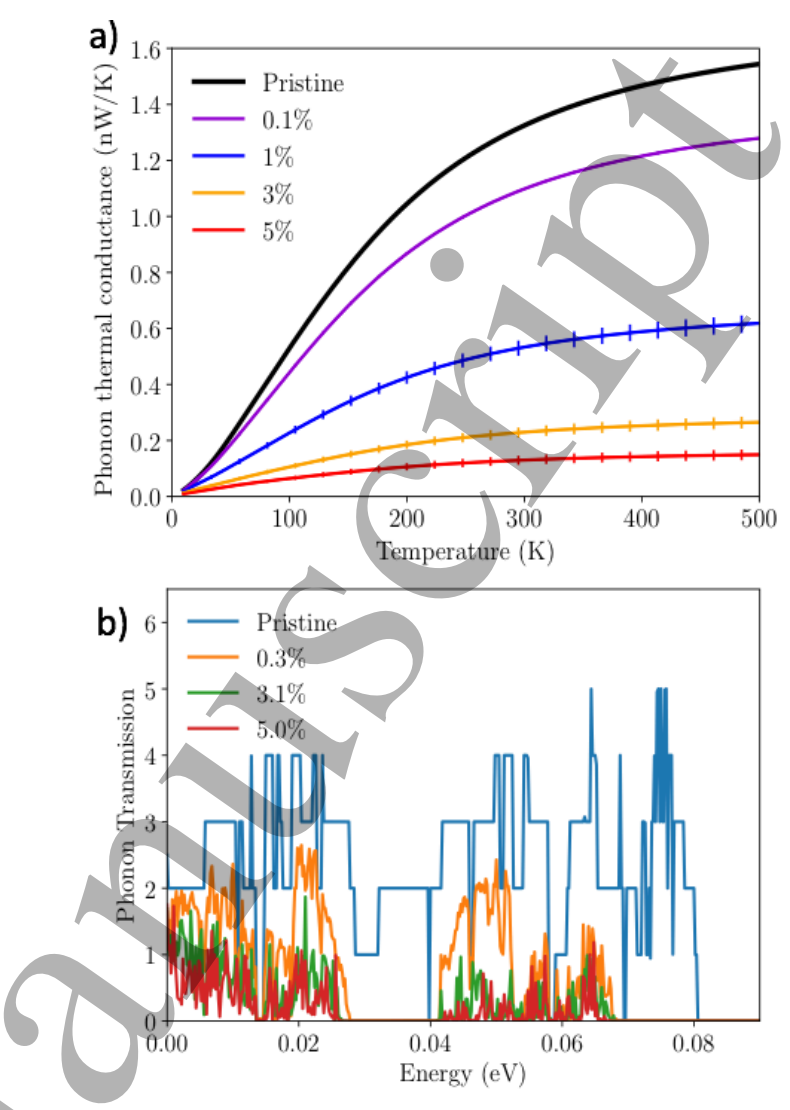

FIG. 3. a) Average phonon thermal conductance as a function of temperature for an A-SNR of width $N=20$ and length $L \simeq$ $26 \mathrm{~nm}$. These curves correspond to averages over a hundred of random realizations of the disorder. The standard deviation is represented by the error bars. Panel b) phonon transmission probability as a function of the phonon energy and for the different vacancy concentrations $n$.

These results point out that the appearance of a low concentration of randomly distributed vacancies may enhance the thermoelectric behavior of A-SNRs, as we will discuss in the next section.

\section{B. Thermoelectric properties of A-SNRs}

In bulk metals, high values of the figure of merit $Z T$ cannot be obtained because of the Wiedemann-Franz law is generally valid. However, in nanoscale systems, thermal and electronic properties can be tuned independently in order to achieve improved efficiency above this limit. Therefore, the Wiedemann-Franz law may not hold at the nanoscale thanks to interference effects, which in general terms, modify the universal ratio between the electronic contribution to the thermal conductance and the electronic conductance (reflected in the Lorentz number, defined as the ratio of the electric and electron thermal conductivities). Thus, it has been reported $Z T$ 
values greater than 2.4 in tailored nanostructured materials such as superlattices, ${ }^{61}$ nanowires ${ }^{62}$ or quantum dots. ${ }^{63}$ These high efficiencies are obtained by an appropriated combination of phonon suppression and the enhancement of the electronic properties of the device. Therefore, nanostructures made of silicene sheets and silicene nanoribbons seem to be good candidates to exhibits this improved behaviour. ${ }^{51,58}$ In this context, in what follows, we will focus on the thermoelectric response of A-SNRs with vacancy defects. As we discussed above, the random distribution of vacancies largely reduces the phonon thermal conductance of the A-SNR, which is the key ingredient to improve the thermoelectric efficiency of the system.

To analyze the thermoelectric behaviour of the disordered A-SNR, we have adopted the linear response approximation, in which an effective voltage drop $\Delta V$ and a temperature difference $\Delta T$ are applied between the left and right contacts. Within this approach, the electronic current $I_{e}$ and the heat current $I_{Q}$ are given by ${ }^{64}$

$$
\begin{aligned}
I_{e} & =-e^{2} L_{0} \Delta V+\frac{e}{T} L_{1} \Delta T, \\
I_{Q} & =e L_{1} \Delta V-\frac{1}{T} L_{2} \Delta T,
\end{aligned}
$$

where $e$ is the elementary charge, $T$ is the absolute temperature and $L_{n}$ (with $n=0,1,2$ ) are integrals defined as

$$
L_{n}(\mu, T)=\frac{2}{h} \int_{0}^{\infty} \mathcal{T}(E)(E-\mu)^{n}\left(-\frac{\partial f(E, T)}{\partial E}\right) \mathrm{d} E
$$

Here $h$ is the Planck constant, $\mu$ is the chemical potential, $f(E, T)$ is the equilibrium Fermi-Dirac distribution and $\mathcal{T}(E)$ is the energy-dependent electron transmission probability.

The electronic conductance is defined as $\sigma=-I_{e} / \Delta V$ and it can be obtained directly from equation (3)

$$
\sigma(\mu, T)=e^{2} L_{0}
$$

The Seebeck coefficient $S$ is calculated in the linear response regime, namely $|\Delta T| \ll T$ and $|e \Delta V| \ll \mu$. It is defined as the voltage drop induced by a temperature gradient at zero electric current, $S=\Delta V /\left.\Delta T\right|_{I e}=0[$ see equation (3)], in the limit $\Delta T \rightarrow 0$. Thus

$$
S(\mu, T)=-\frac{1}{e T} \frac{L_{1}}{L_{0}}
$$

The electron contribution to the thermal conductance is defined as the ratio between the thermal current $I_{Q}$ and the temperature gradient $\Delta T$ when the electric current $I_{e}$ is zero, $\kappa_{\mathrm{el}}=I_{Q} /\left.\Delta T\right|_{I_{e}=0}$. Written in terms of the integrals (5), it is given by

\footnotetext{
The total thermal conductance of the SNRs is obtained as $\kappa(\mu, T)=\kappa_{\mathrm{el}}(\mu, T)+\kappa_{\mathrm{ph}}(T)$. It should be stressed
}

that $\kappa_{\mathrm{ph}}(T)$ depends only on temperature but not on the chemical potential $\mu$. Finally, the thermoelectric efficiency is determined by the figure of merit

$$
Z T(\mu, T)=\frac{\sigma S^{2} T}{\kappa_{\mathrm{el}}(\mu, T)+\kappa_{\mathrm{ph}}(T)} .
$$

In Fig. 4 we show the results of the average values (over a hundred of realizations) of the electron conductance, the Seebeck coefficient, the total thermal conductance $\left\langle\kappa_{\text {total }}\right\rangle=\left\langle\kappa_{\mathrm{ph}}\right\rangle+\left\langle\kappa_{\mathrm{el}}\right\rangle$, the figure of merit and the Lorentz number of the disordered SNR, for a fixed temperature of $300 \mathrm{~K}$. These plots show the different thermoelectric quantities for pristine (black lines) and the disordered A-SNRs, with vacancy concentration of $1 \%$ (violet lines), $3 \%$ (light-green lines), $4 \%$ (mustardyellow lines) and 5\% (red lines). The electronic conductance and the total thermal conductance as a function of the chemical potential [Fig. 4 (a) and (b)] exhibit a marked reduction at the center of the band $(\mu=0)$ as the concentration of vacancies increases. For instance, for concentrations of $1 \%$ and $3 \%$, the electronic conductance decreases about $45 \%$ and $80 \%$, respectively. The occurrence of Anderson electron localization ${ }^{65}$ explains the overall decrease of the electronic conductance due to the existence of a disordered potential landscape caused by vacancies. We observed similar results in a previous work, where we calculated the electron localization length $\lambda_{e}$ for different vacancy concentration. ${ }^{66}$ In the case of the total thermal conductance, the combination of the reduction of the phonon mean-free-path due to phonon-defect scattering and the Anderson electron localization (which affects the electron transmission function and consequently the electronic contribution to the thermal conductance) generate the remarkable reduction of $\left\langle\kappa_{\text {total }}\right\rangle$. On the other hand, due to the breaking of electron-hole symmetry and the destructive quantum interference effects within the conductor (Fano-like effect), the Seebeck coefficient displays an enhancement of the absolute maximum values as the vacancy concentration increases, as shown in Fig. 4(c). The Seebeck coefficient is sensitive to abrupt changes in the transmission function $\mathcal{T}(E)$ because, in the first order of approximation, it depends directly on the derivative of $\mathcal{T}(E)$ with respect to the energy, known as Mott's formula. ${ }^{64,67}$ Thus, the sharp reduction of the transmission probability through the disordered conductor (which is very similar to the conductance behavior) explains the enhancement of the Seebeck coefficient. Finally, the figure of merit $Z T$ does not show a monotonic trend but reaches the highest values at a concentration of $3 \%$ [Fig. 4 (d)], which corresponds to an optimal combination of the physical properties that determine the thermoelectric efficiency. We calculate the Lorentz number for different vacancy concentrations, averaged over a hundred of random configurations. The average Lorentz number decreases as the vacancy concentration increases. It is due to the reduction of the electronic contribution to the thermal conductance. The faster reduction of the thermal conductance 
on increasing the magnitude of disorder compared to the electric conductance can be understood as follows. The disorder has stronger impact on electron states of higher energy, that become more spatially localized (Anderson localization) due to their smaller wavelength. Therefore, electron transmission at high energy is dramatically reduced while it is less affected at low energy. This change in transmission compared to the pristine nanoribbon results in a decrease of $L_{0}$ (see equation 5 ) and then a reduction of $\sigma$ is expected. However, the reduction of $L_{2}$ is more pronounced due to the weighting factor $(E-\mu)^{2}$ in the integral appearing in equation 5 . Hence, the ratio $L_{2} / L_{0}$ (i.e. the Lorenz number) decreases with disorder, as observed in Fig. 4 (e).
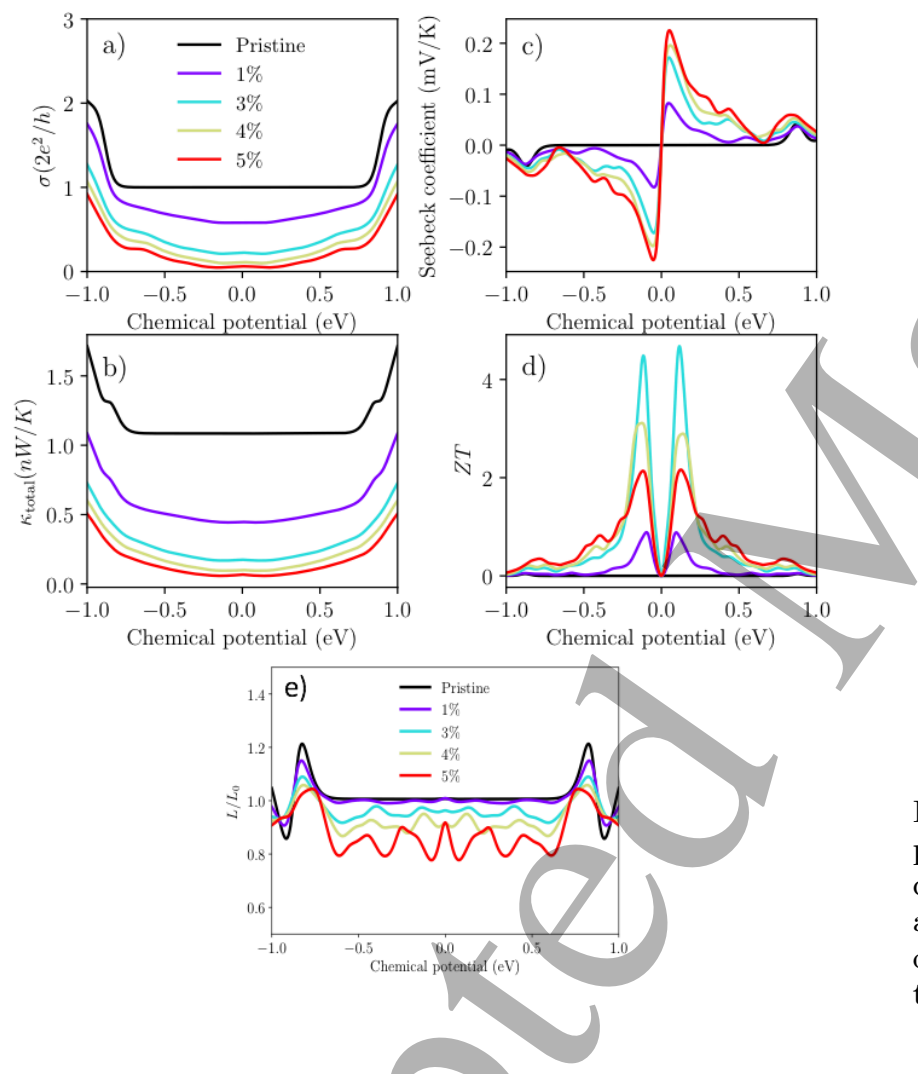

FIG. 4. (a) Electronic conductance, (b) total thermal conductance, (c) Seebeck coefficient, (d) figure of merit and (e) Lorentz number as a function of the chemical potential $\mu$, for a fixed temperature $T=300 \mathrm{~K}$. Results are shown for a pristine sample and disordered samples with vacancy concentrations of $1 \%, 3 \%, 4 \%$ and $5 \%$.

We can gain insight into the above result by plotting the maximum of the figure of merit, $Z T_{\max }$, as a function of temperature, as shown in Fig. 5(a). Again, $Z T_{\max }$ reaches its highest value at a vacancy concentration of $3 \%$. The behaviour of $Z T_{\max }$ can be explained by the interplay between $\kappa_{\mathrm{el}}, \kappa_{\mathrm{ph}}$ and $S$. In our system, we have confirmed that the Wiedemann-Franz law holds to a large extend, i.e., $\kappa_{\mathrm{el}} / \sigma T \simeq\left(\pi^{2} / 3\right)\left(k_{B} / e\right)^{2}$. Thus

$$
Z T \simeq \frac{3}{\pi^{2}}\left(\frac{e}{k_{B}}\right)^{2} \frac{S^{2}}{1+\kappa_{\mathrm{ph}} / \kappa_{\mathrm{el}}}
$$

In Fig. $5(\mathrm{~b})$, we plot $\kappa_{\mathrm{ph}} / \kappa_{\mathrm{el}}$ as a function of the chem-

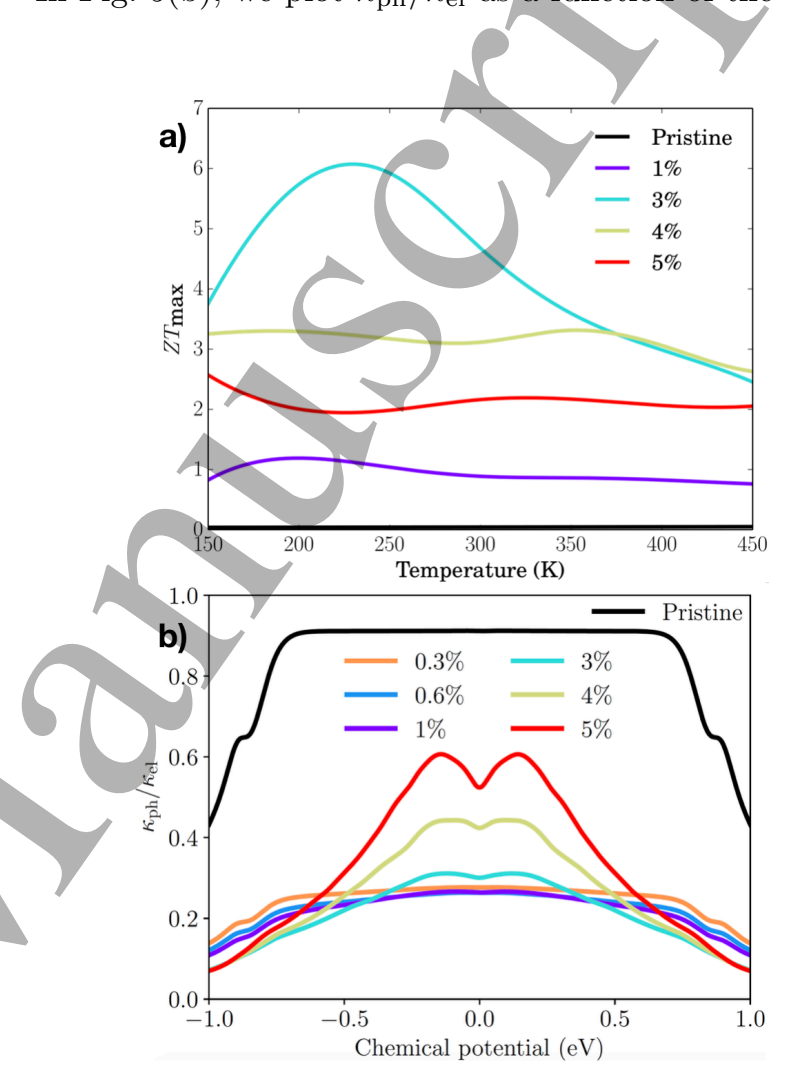

FIG. 5. a) Maximum figure of merit as a function of temperature for concentrations of $1 \%, 3 \%, 4 \%$ and $5 \%$. As a comparison, the pristine case is also shown; b) Ratio $\kappa_{\mathrm{ph}} / \kappa_{\mathrm{el}}$ as a function of the chemical potential at $T=300 \mathrm{~K}$ for various values of the concentration of vacancies. For comparison, the result for the prinstine SNR is also shown (black line).

ical potential at $T=300 \mathrm{~K}$ for various values of the concentration of vacancies. We can see that the maximum of $\kappa_{\mathrm{ph}} / \kappa_{\mathrm{el}}$ decreases with the concentration of vacancies, provided that the concentration does not exceed $\sim 1 \%$. The opposite behavior is observed at larger concentrations. As the maximum of $\kappa_{\mathrm{ph}} / \kappa_{\mathrm{el}}$ begins to grow faster than $S^{2}, Z T$ decreases with the concentration of vacancies. Our simulations indicate that the optimum concentration of vacancies is about 3\%. Larger values of the concentration worsen the thermoelectric efficiency of the A-SNRs. It is worth noticing that for the whole range of the concentration of vacancies studied in this work $\kappa_{\mathrm{ph}}<\kappa_{\mathrm{el}}$. Therefore, vacancy defects efficiently reduce the lattice thermal conductance while electron thermal transport is less affected. 


\section{CONCLUSIONS}

In this work, we have investigated the thermoelectric properties of silicene nanoribbons in the presence of a random distribution of atomic vacancies in a diluted regime. We have obtained the phonon and electron contribution to the thermal conductance, the electronic conductance, the Seebeck coefficient, the figure of merit, and the Lorentz number of these nanoribbons. Due to the phonon-defect scattering within the conductor, which suppresses some optical and acoustic vibrational modes, we have found a significant decrease of the phonon thermal conductance as a function of the defect concentration, over a wide range of temperature. Besides, due to the break of electron-hole symmetry and the destructive quantum interference effects within the conductor, we have obtained an enhancement of the Seebeck coefficient as the vacancy concentration is increased, reaching an asymptotic value at concentrations above $5 \%$. We have observed that the figure of merit $Z T_{\max }$ exhibits a maximum behavior for a concentration of $3 \%$, which corresponds to an optimal value of the factor $S^{2} / \kappa_{\text {total }}$ in the diluted regime. Finally, the ratio between the electron and the phonon thermal conductances reaches an optimum value for a specific concentration, leading to an overall remarkable thermoelectric efficiency. With the above findings, we can conclude that the addition of vacancies is a suitable method for designing better and more efficient nanoscale thermoelectric devices based on silicene.

\section{ACKNOWLEDGMENTS}

P.A.O and L.R. thank the financial support by FONDECYT (Grant No. 1180914) and DGIIP-USM grant. Work in Madrid was supported by MINECO (Grant MAT2016-75955). C.N. thanks a scholarship from CONICYT-Chile and the DGIIP for financial support by PIIC grant.
${ }^{1} \mathrm{H}$. J. Goldsmid, Introduction to thermoelectricity (Springer, Berlin, 2010).

2 C. Villagonzalo, R. Römer, and M. Schreiber, Eur. Phys. J. B 12 (1999).

3 C. Gayner and K. K. Kar, Prog. Mater. Sci. 83, 330 (2016).

4 F. Domínguez-Adame, M. Martín-González, D. Sánchez, and A. Cantarero, Physica E 113, 213 (2019).

${ }^{5}$ L. D. Hicks and M. S. Dresselhaus, Phys. Rev. B 47, 16631 (1993).

6 A. Khitun, A. Balandin, J. L. Liu, and K. L. Wang, J. Appl. Phys. 88, 696 (2000).

7 A. A. Balandin and O. L. Lazarenkova, Appl. Phys. Lett. 82, 415 (2003).

${ }^{8}$ H. Sadeghi, S. Sangtarash, and C. J. Lambert, Beilstein J. Nanotechnol. 6, 1176 (2015).

9 R. Venkatasubramanian, E. Siivola, T. Colpitts, and B. O'Quinn, Nature (London) 413, 597 (2001).

10 T. C. Harman, P. J. Taylor, M. P. Walsh, and B. E. LaForge, Science 297, 2229 (2002).

11 A. I. Hochbaum, R. Chen, R. D. Delgado, W. Liang, E. C. Garnett, M. Najarian, A. Majumdar, and P. Yan, Nature (London) 451, 163 (2008).

12 A. I. Boukai, Y. Bunimovich, J. Tahir-Kheli, J.-K. Yu, W. A. Goddard, and J. R. Heath, Nature (London) 451, 168 (2008).

13 G. D. Mahan and J. O. Sofo, Proc. Natl. Acad. Sci. USA 93, 7436 (1996).

14 G. Gómez-Silva, O. Ávalos-Ovando, M. L. Ladrón de Guevara, and P. A. Orellana, J. Appl. Phys. 111, 053704 (2012).

15 J. Zheng, M.-J. Zhu, and F. Chi, J. Low Temp. Phys. 166, 208 (2012).

16 V. M. García-Suárez, R. Ferradás, and J. Ferrer, Phys. Rev. B 88, 235417 (2013).

17 H.-H. Fu, L, Gu, D.-D. Wu, and Z.-Q. Zhang, Phys. Chem. Chem. Phys. 17, 11077 (2015).
18 M. A. Sierra, M. Saiz-Bretín, F. Domínguez-Adame, and D. Sánchez, Phys. Rev. B 93, 235452 (2016).

19 R.-N. Wang, G.-Y. Dong, S.-F. Wang, G.-S. Fu, and J.-L. Wang, J. Appl. Phys. 120, 184303 (2016).

20 L. Yang, Z.-G. Chen, M. Hong, G. Han, and J. Zou, ACS Appl. Mater. Interfaces 7, 23694 (2015).

21 K. Woochul, J. Mater. Chem. C 3, 10336 (2015).

22 S. Xiong and S. Volz, C. R. Phys 17, 1146 (2016).

23 M. Saiz-Bretín, A. V. Malyshev, F. Domínguez-Adame, D. Quigley, and R. A. Römer, Carbon 127, 64 (2018).

${ }^{24}$ K. Koumoto and T. Mori, Thermoelectric nanomaterials. Materials design and applications (Springer, Berlin, 2013).

25 M. Saiz-Bretín, A. V. Malyshev, P. A. Orellana, and F. Domínguez-Adame, Phys. Rev. B 91, 085431 (2015).

26 A. V. Savin, Y. S. Kivshar, and B. Hu, Phys. Rev. B 82, 195422 (2010).

27 J. Hu, S. Schiffli, A. Vallabhaneni, X. Ruan, and Y. P. Chen, Appl. Phys. Lett. 97, 133107 (2010).

28 F. Mazzamuto, V. Hung Nguyen, Y. Apertet, C. Car, C. Chassat, J. Saint-Martin, and P. Dollfus, Phys. Rev. B 83, 235426 (2011).

29 K.-M. Li, Z.-X. Xie, K.-L. Su, W.-H. Luo, and Y. Zhang, Phys. Lett. A 378, 1383 (2014).

30 H.-S. Zhang, Z.-X. Guo, X.-G. Gong, and J.-X. Cao, J. Appl. Phys. 112, 123508 (2012).

${ }^{31}$ Y. Chen, T. Jayasekera, A. Calzolari, K. W. Kim, and M. Buongiorno Nardelli, J. Phys.: Condens. Matter 22, $372202(2010)$.

${ }^{32}$ Y. Xu, X. Chen, J.-S. Wang, B.-L. Gu, and W. Duan, Phys. Rev. B 81, 195425 (2010).

33 E. Pop, V. Varshney, and A. K. Roy, MRS Bulletin 37, 1273 (2012).

${ }^{34}$ Hai-peng Li and Rui-qin Zhang, EPL (Europhys. Lett.) 99, 36001 (2012).

${ }^{35}$ L. C. Lew Yan Voon, J. Zhu, and U. Schwingenschlögl, Appl. Phys. Rev. 3, 040802 (2016). 
36 B. Aufray, A. Kara, S. Vizzini, H. Oughaddou, C. Landri, B. Ealet, and G. Le Lay, Appl. Phys. Lett. 96, 183102 (2010).

37 B. Lalmi, H. Oughaddou, H. Enriquez, A. Kara, S. Vizzini, B. Ealet, and B. Aufray, Appl. Phys. Lett. 97, 223109 (2010).

38 M. Ezawa, New J. Phys. 14, 033003 (2012).

39 J. Zhao, H. Liu, Z. Yu, R. Quhe, S. Zhou, Y. Wang, C. C. Liu, H. Zhong, N. Han, J. Lu, Y. Yao, and K. Wu, Prog. Mater. Sci. 83, 24 (2016).

40 Y. Ding and J. Ni, Appl. Phys. Lett. 95, 083115 (2009).

41 S. Cahangirov, M. Topsakal, E. Aktürk, H. Şahin, and S. Ciraci, Phys. Rev. Lett. 102, 236804 (2009).

42 Y. Ouyang and J. Guo, Appl. Phys. Lett. 94, 263107 (2009).

43 D. Gunlycke, H. Lawler, and C. White, Phys. Rev. B 75, 085418 (2007).

${ }^{44}$ F. H. Stillinger and T. A. Weber, Phys. Rev. B 31, 5262 (1985).

45 S. Das and A. Dhar, Eur. Phys. J. B 85, 372 (2012).

46 J.-S. Wang, N. Zeng, J. Wang, and C. K. Gan, Phys. Rev. E 75, 061128 (2007).

47 T. Yamamoto and K. Watanabe, Phys. Rev. Lett. 96, 255503 (2006).

48 J. Schneider, J. Hamaekers, S. T. Chill, S. Smidstrup, R. T. J. Bulin, A. Blom, and K. Stokbro, Model. Simul. Mater. Sci. Eng. 25, 085007 (2017).

49 K. Yang, S. Cahangirov, A. Cantarero, A. Rubio, and R. D'Agosta, Phys. Rev. B 89, 125403 (2014).

50 Z. Wang, T. Feng, and X. Ruan, J. Appl. Phys. 117, 084317 (2015).

${ }^{51}$ K. Zberecki, M. Wierzbicki, J. Barna, and R. Swirkowicz, Phys. Rev. B 88, 115404 (2013).

${ }^{52}$ F. Hao, D. Fang, and Z. Xu, Appl. Phys. Lett. 99, 041901 (2011).
53 L. J. Wirth, T. H. Osborn, and A. A. Farajian, Appl. Phys. Lett. 109, 173102 (2016).

54 S. Li, Y. Wu, Y. Tu, Y. Wang, T. Jiang, W. Liu, and Y. Zhao, Sci. Rep. 5, 7881 (2015).

55 V. O. Özçelik, H. H. Gurel, and S. Ciraci, Phys. Rev. B 88, 045440 (2013).

56 W. Zhao, Z. X. Guo, J. X. Cao, and J. W. Ding, AIP Advances 1, 042135 (2011).

57 M. Hu, X. Zhang, K. P. Giapis, and D. Poulikakơs, Phys. Rev. B 84, 085442 (2011).

58 L. Pan, H. J. Liu, X. J. Tan, H. Y. Lv, J. Shi, X. F. Tang, and G. Zheng, Phys. Chem. Chem. Phys. 14, 13588 (2012).

59 C. A. Polanco and L. Lindsay, Phys. Rev. B 97, 014303 (2018)

60 M. Barati1, T. Vazifehshenas, T. Salavati-fard, and M. Farmanbar, J. Phys.: Condens. Matter 30, 155307 (2018)

61 R. Venkatasubramanian, E. Siivola, T. Colpitts, and B. O'Quinn, Nature (London) 413, 597 (2001).

62 A. I. Hochbaum, R. Chen, R. D. Delgado, W. Liang, E. C. Garnett, M. Najarian, A. Majumdar, and Yang, Nature (London) 451, 163 (2008).

63 T. C. Harman, P. J. Taylor, M. P. Walsh, and B. E. LaForge, Science 297, 2229 (2002).

${ }^{64}$ U. Sivan and Y. Imry, Phys. Rev. B 33, 551 (1986).

65 E. Abrahams, P. W. Anderson, D. C. Licciardello, and T. V. Ramakrishnan, Phys. Rev. Lett. 42, 673 (1979).

66 C. Núnez, F. Domínguez-Adame, P. A. Orellana, L. Rosales, and R. A. Römer, 2D Materials 3, 025006 (2016).

67 N. F. Mott and H. Jones, The Theory of the Properties of Metalsand Alloys (Dover Publications, New York, 1958). 